\title{
Stochastic Comparisons of the Smallest Claim Amounts from Two Sets of Independent Portfolios
}

\author{
Hossein Nadeb \\ Yazd University, Yazd, Iran
}

\author{
Hamzeh Torabi
}

Yazd University, Yazd, Iran

\begin{abstract}
The aim of this paper is detecting the ordering properties of the smallest claim amounts arising from two sets of independent heterogeneous portfolios in insurance. First, we prove a general theorem that it presents some sufficient conditions in the sense of the hazard rate ordering to compare the smallest claim amounts from two batches of independent heterogeneous portfolios. Then, we show that the exponentiated scale model as a famous model and the Harris family satisfy the sufficient conditions of the proven general theorem. Also, to illustrate our results, some used models in actuary are numerically applied.
\end{abstract}

Keywords: hazard function, majorization, smallest claim amounts, stochastic ordering.

\section{Introduction}

Let $X_{1}, \ldots, X_{n}$ be a set of independent and non-negative random variables such that $X_{i}$, $i=1, \ldots, n$, corresponds to the hazard rate function $r\left(x ; \alpha_{i}, \beta_{i}\right)$. Suppose that $X_{i}$ denotes the total random losses faced by the $i$ th policyholder in an insurance period, which is called the $i$ th claim severity. Also, suppose that $I_{p_{1}}, \ldots, I_{p_{n}}$ are independent Bernoulli random variables independent of $X_{i}$ 's, with $\mathrm{E}\left[I_{p_{i}}\right]=p_{i}, i=1, \ldots, n$; which $I_{p_{i}}=1$ whenever the $i$ th policyholder makes random claim amount $X_{i}$ and $I_{p_{i}}=0$ whenever does not make a claim. In this setting, $Y_{i}=I_{p_{i}} X_{i}$ is a claim amount corresponding to $i$ th policyholder and $\left(Y_{1}, \ldots, Y_{n}\right)$ is said to be a portfolio of risks. In this respect, the parameters corresponding to this portfolio are $\alpha_{1}, \ldots, \alpha_{n}, \beta_{1}, \ldots, \beta_{n}$ and $p_{1}, \ldots, p_{n}$. Further, consider another portfolio of risks $\left(Y_{1}^{*}, \ldots, Y_{n}^{*}\right)$ with other corresponding parameters $\alpha_{1}^{*}, \ldots, \alpha_{n}^{*}, \beta_{1}^{*}, \ldots, \beta_{n}^{*}$ and $p_{1}^{*}, \ldots, p_{n}^{*}$.

The annual premium is the primary cost to the policyholder for assigning the risk to the insurer which depends on the type of insurance. Determination of the annual premium is an important purpose in insurance analysis. To determine the annual premium, some quantities such as the number of claims, the aggregate claim amounts and the smallest and largest claim amounts play important role.

The discovery of preferences between random future gains or losses is an interesting problem for the actuaries. For this target, stochastic orderings are very helpful. They have been extensively used in some areas of sciences such as management science, financial economics, insurance, actuarial science, operation research, reliability theory, queuing theory and survival 
analysis. Many stochastic orderings to compare the random variables have been introduced in the literature. These orderings consider the comparisons of the various properties of the random variables. Some stochastic orderings compare the location or the magnitude of random variables such as the usual, the hazard rate, the reversed hazard rate, the mean residual life and the likelihood ratio orderings. Some stochastic orderings compare the variability or the dispersion of random variables such as the convex and the dispersive orderings. Some of them are according to compare the location and the spread orderings such as the increasing convex and the increasing concave orderings. For a comprehensive discussion on the various stochastic orderings, we refer to Müller and Stoyan (2002), Li and Li (2013) and Shaked and Shanthikumar (2007).

The problem of stochastic comparisons of some important functions in $\left(Y_{1}, \ldots, Y_{n}\right)$ and $\left(Y_{1}^{*}, \ldots, Y_{n}^{*}\right)$, such as the number of claims, $\sum_{i=1}^{n} I_{p_{i}}$, the aggregate claim amounts, $\sum_{i=1}^{n} Y_{i}$, the smallest, $Y_{1: n}=\min \left(Y_{1}, \ldots, Y_{n}\right)$, and the largest claim amounts, $Y_{n: n}=\max \left(Y_{1}, \ldots, Y_{n}\right)$ in two portfolios, have been considered by many researchers. For instance, Karlin and Novikoff (1963) worked on the number of claims and Ma (2000), Frostig (2001), Hu and Ruan (2004), Denuit and Frostig (2006), Khaledi and Ahmadi (2008), Zhang and Zhao (2015), Barmalzan, Najafabadi, and Balakrishnan (2015), Li and Li (2016) and Barmalzan, Najafabadi, and Balakrishnan (2018) worked on the aggregate claim amounts.

Over the past few years, the comparisons of the smallest and largest claim amounts arising from two sets of heterogeneous portfolios have been more considered.

Balakrishnan, Zhang, and Zhao (2018) investigated the comparisons of the largest and the sample range of claim amounts arising from two sets of heterogeneous portfolios in the sense of the usual, the hazard rate and the reversed hazard rate orderings, when the severities in portfolios are independent.

Nadeb, Torabi, and Dolati (2020b) worked on the comparisons of the largest claim amounts in two heterogeneous portfolios in the sense of the usual stochastic ordering, when the existing severities in each portfolio are dependent.

Barmalzan, Najafabadi, and Balakrishnan (2017) discussed on ordering the largest and smallest claim amounts in the sense of the usual stochastic ordering and the hazard rate ordering when the severities belong to the scale model.

Barmalzan and Najafabadi (2015) discussed on the stochastic comparisons between the smallest claim amounts in two portfolios in the sense of the convex transform ordering, which is a well-known criterion to compare the skewness and coefficient of variations of probability distributions, and right-spread ordering whenever the severities follow the Weibull distribution.

Barmalzan, Najafabadi, and Balakrishnan (2016) considered the Weibull distribution as the distribution of severities and discussed on the stochastic comparisons between the smallest claim amounts in two portfolios in the sense of the usual stochastic ordering, the hazard rate ordering and the likelihood ratio ordering.

Recently, Nadeb, Torabi, and Dolati (2020a) considered the transmuted-G model as a suitable model for severities in insurance analysis and worked on the stochastic comparisons between the largest and smallest claim amounts in two portfolios in the sense of the usual, reversed hazard rate and hazard rate orderings.

Motivated by the importance of the smallest claim amounts and some of the articles mentioned above, we concentrate on the stochastic comparisons of the smallest claim amounts from two sets of independent heterogeneous portfolios. The current paper provides the hazard rate ordering, which compares the magnitude of the random variables, to compare the smallest claim amounts in two portfolios related to the insurance companies.

The rest of the paper is organized as follows. In Section 2, we recall some definitions and lemmas which are useful to prove our main results. In Section 3, stochastic comparisons of the smallest claim amounts from two independent heterogeneous portfolios of risks in a general model in the sense of the hazard rate ordering are studied. Some numerical examples are also 
illustrated to observe the validity of the results.

\section{The basic definitions and some prerequisites}

In this section, we recall some definitions and useful lemmas which are helpful to prove the main results. Throughout the paper, we use the notations $\mathbb{R}=(-\infty,+\infty)$ and $r_{X}(x)$ for the hazard function corresponding to the random variable $X$.

Let $X$ and $Y$ be two non-negative random variables with the hazard rate functions $r_{X}($.$) and$ $r_{Y}($.$) , respectively. In the following, we state the definition of the hazard rate ordering.$

Definition 1. The random variable $X$ is said to be smaller than $Y$ in the hazard rate ordering (denoted by $X \leq_{\mathrm{hr}} Y$ ) if $r_{X}(x) \geq r_{Y}(x)$ for all $x \in \mathbb{R}$.

All results of this paper deal with comparison of two hazard rate functions in the basis of comparing the parameter vectors or matrices. To compare the parameter vectors or matrices, we use the concepts of majorization of vectors and matrices. In the following, we state the concepts of majorization and weak majorization for comparing the vectors. We use the notation $x_{1: n} \leq x_{2: n} \leq \ldots \leq x_{n: n}$ to denote the increasing arrangement of the components of a vector $\boldsymbol{x}=\left(x_{1}, \ldots, x_{n}\right)$.

Definition 2. The vector $\boldsymbol{x}$ is said to be

(i) weakly submajorized by the vector $\boldsymbol{y}$ (denoted by $\boldsymbol{x} \preceq_{\mathrm{w}} \boldsymbol{y}$ ) if $\sum_{i=j}^{n} x_{i: n} \leq \sum_{i=j}^{n} y_{i: n}$ for all $j=1, \ldots, n$,

(ii) weakly supermajorized by the vector $\boldsymbol{y}$ (denoted by $\boldsymbol{x} \preceq \boldsymbol{w}$ y) if $\sum_{i=1}^{j} x_{i: n} \geq \sum_{i=1}^{j} y_{i: n}$ for all $j=1, \ldots, n$,

(iii) majorized by the vector $\boldsymbol{y}$ (denoted by $\boldsymbol{x} \preceq \boldsymbol{m}$ ) if $\sum_{i=1}^{n} x_{i}=\sum_{i=1}^{n} y_{i}$ and $\sum_{i=1}^{j} x_{i: n} \geq$ $\sum_{i=1}^{j} y_{i: n}$ for all $j=1, \ldots, n-1$.

The concepts of majorization and weak majorization were provided in the previous definition in order to compare two numerical vectors. The following lemma provides the comparison of two multivariate real-valued function in the basis of comparison of the input values of the function.

Lemma 1 (Marshall, Olkin, and Arnold (2011), Theorem 3.A.7). Let $\phi$ be a continuous real valued function on the set $\mathscr{D}=\left\{\boldsymbol{x}: x_{1} \geq x_{2} \geq \ldots \geq x_{n}\right\}$ and continuously differentiable on the interior of $\mathscr{D}$. Denote the partial derivative of $\phi$ with respect to $i$ th argument by $\phi_{(i)}(\boldsymbol{z})=\partial \phi(\boldsymbol{z}) / \partial z_{i}$. Then,

$$
\phi(\boldsymbol{x}) \leq \phi(\boldsymbol{y}) \quad \text { whenever } \boldsymbol{x} \preceq_{\mathrm{w}} \boldsymbol{y} \text { on } \mathscr{D}
$$

if and only if

$$
\phi_{(1)}(\boldsymbol{z}) \geq \phi_{(2)}(\boldsymbol{z}) \geq \ldots \geq \phi_{(n)}(\boldsymbol{z}) \geq 0
$$

i.e. the gradient $\nabla \phi(\boldsymbol{z}) \in \mathscr{D}_{+}=\left\{\boldsymbol{x}: x_{1} \geq x_{2} \geq \ldots \geq x_{n} \geq 0\right\}$, for all $\boldsymbol{z}$ in the interior of $\mathscr{D}$. Similarly,

$$
\phi(\boldsymbol{x}) \leq \phi(\boldsymbol{y}) \quad \text { whenever } \quad \stackrel{\boldsymbol{x}}{\mathfrak{w}} \boldsymbol{y} \text { on } \mathscr{D}
$$

if and only if

$$
0 \geq \phi_{(1)}(\boldsymbol{z}) \geq \phi_{(2)}(\boldsymbol{z}) \geq \ldots \geq \phi_{(n)}(\boldsymbol{z})
$$

i.e. the gradient $\nabla \phi(\boldsymbol{z}) \in \mathscr{D}_{-}=\left\{\boldsymbol{x}: 0 \geq x_{1} \geq x_{2} \geq \ldots \geq x_{n}\right\}$, for all $\boldsymbol{z}$ in the interior of $\mathscr{D}$. 
To compare two real-valued functions that their input values are matrices, we need to some concepts to compare the matrices. Therefore, we firstly recall the concepts of permutation matrix, $T$-transform matrix and doubly stochastic matrix, and then state the multivariate majorization of matrices.

Definition 3. A square matrix

(i) is called a permutation matrix if each row and each column has a single unit, and all other entries are zero;

(ii) is called a $T$-transform matrix if is of the form $T_{\omega}=\omega I_{n}+(1-\omega) \Pi$, where $0 \leq \omega \leq 1$, $I_{n}$ is an $n \times n$ identity matrix and $\Pi$ is a permutation matrix that just interchanges two coordinates;

(iii) $P=\left[p_{i j}\right]$ is called a doubly stochastic matrix if $p_{i j} \geq 0$, for $i, j=1, \ldots, n, \sum_{i=1}^{n} p_{i j}=$ $1, j=1, \ldots, n$, and $\sum_{j=1}^{n} p_{i j}=1, i=1, \ldots, n$.

Now, we state the concepts of majorization for comparing the matrices.

Definition 4. Let $A=\left[a_{i j}\right]$ and $B=\left[b_{i j}\right]$ be two $m \times n$ matrices with the rows $\boldsymbol{a}_{1}^{R}, \ldots, \boldsymbol{a}_{m}^{R}$ and $\boldsymbol{b}_{1}^{R}, \ldots, \boldsymbol{b}_{m}^{R}$, respectively. Then

(i) $A$ is said to be chain majorized by $B$, denoted by $A \ll B$, if there exists a finite set of $n \times n T$-transform matrices $T_{\omega_{1}}, \ldots, T_{\omega_{k}}$, such that $A=B T_{\omega_{1}} \times \ldots \times T_{\omega_{k}}$;

(ii) $A$ is said to be majorized by $B$, denoted by $A<B$, if there exist a $n \times n$ doubly stochastic matrix $P$, such that $A=B P$;

(iii) $A$ is said to be row majorized by $B$, denoted by $A \stackrel{\text { row }}{<} B$, if $\boldsymbol{a}_{i}^{R} \preceq \boldsymbol{b}_{i}^{R}$, for $i=1, \ldots, m$;

(iv) $A$ is said to be row weakly submajorized by $B$, denoted by $A \underset{\mathrm{w}}{<} B$, if $\boldsymbol{a}_{i}^{R} \preceq_{\mathrm{w}} \boldsymbol{b}_{i}^{R}$, for $i=1, \ldots, m$.

The following implications are hold between the different types of majorization of matrices:

$$
A \ll B \Rightarrow A<B \Rightarrow A \stackrel{\text { row }}{<} B \Rightarrow A \underset{\mathrm{w}}{<} B
$$

For more details on the mentioned topics, one may refer to Marshall et al. (2011).

Also, we define two required spaces as the following which are used in the paper.

$$
\begin{aligned}
& \mathscr{S}_{n}^{-}=\left\{(\boldsymbol{x}, \boldsymbol{y})=\left[\begin{array}{l}
x_{1} \ldots x_{n} \\
y_{1} \ldots y_{n}
\end{array}\right]:\left(x_{i}-x_{j}\right)\left(y_{i}-y_{j}\right) \leq 0, \quad i, j=1, \ldots, n\right\}, \\
& \mathscr{S}_{n}^{+}=\left\{(\boldsymbol{x}, \boldsymbol{y})=\left[\begin{array}{l}
x_{1} \ldots x_{n} \\
y_{1} \ldots y_{n}
\end{array}\right]:\left(x_{i}-x_{j}\right)\left(y_{i}-y_{j}\right) \geq 0, \quad i, j=1, \ldots, n\right\} .
\end{aligned}
$$

\section{Main results}

In this section, the smallest claim amounts from two independent heterogeneous portfolios of risks in the sense of the hazard rate ordering are compared. Some examples are also presented for illustration the validity of the results.

The following theorem provides a comparison between the smallest claim amounts in two independent heterogeneous portfolios of risks for a general model. 
Theorem 1. Let $X_{1}, \ldots, X_{n}\left(X_{1}^{*}, \ldots, X_{n}^{*}\right)$ be independent and non-negative random variables with $X_{i} \sim r\left(x ; \alpha_{i}, \beta_{i}\right)\left(X_{i}^{*} \sim r\left(x ; \alpha_{i}^{*}, \beta_{i}\right)\right), i=1, \ldots, n$. Further, suppose that $I_{p_{1}}, \ldots, I_{p_{n}}$ $\left(I_{p_{1}^{*}}, \ldots, I_{p_{n}^{*}}\right)$ is a set of independent Bernoulli random variables, independent of the $X_{i}$ 's $\left(X_{i}^{*}, \mathrm{~s}\right)$, with $\mathrm{E}\left[I_{p_{i}}\right]=p_{i}\left(\mathrm{E}\left[I_{p_{i}^{*}}\right]=p_{i}^{*}\right), i=1, \ldots, n$. Assume that the following conditions hold:

(i) $r(x ; \alpha, \beta)$ is increasing [decreasing] and convex in $\alpha$ for any $\beta$ and any $x>0$;

(ii) $\frac{\partial r(x ; \alpha, \beta)}{\partial \alpha}$ is decreasing (increasing) in $\beta$ for any $\alpha$ and any $x>0$.

Then, for $(\boldsymbol{\alpha}, \boldsymbol{\beta}) \in \mathscr{S}_{n}^{-}\left(\mathscr{S}_{n}^{+}\right)$and $\left(\boldsymbol{\alpha}^{*}, \boldsymbol{\beta}\right) \in \mathscr{S}_{n}^{-}\left(\mathscr{S}_{n}^{+}\right)$, we have

$$
\prod_{i=1}^{n} p_{i}^{*} \leq \prod_{i=1}^{n} p_{i} \text { and }\left(\alpha_{1}, \ldots, \alpha_{n}\right) \preceq_{\mathrm{w}}\left[\preceq^{\complement}\right]\left(\alpha_{1}^{*}, \ldots, \alpha_{n}^{*}\right) \Longrightarrow Y_{1: n}^{*} \leq_{\mathrm{hr}} Y_{1: n}
$$

Proof. Without loss of generality, assume that $\beta_{1} \leq \ldots \leq \beta_{n}\left(\beta_{1} \geq \ldots \geq \beta_{n}\right)$. For $(\boldsymbol{\alpha}, \boldsymbol{\beta}) \in$ $\mathscr{S}_{n}^{-}\left(\mathscr{S}_{n}^{+}\right)$and $\left(\boldsymbol{\alpha}^{*}, \boldsymbol{\beta}\right) \in \mathscr{S}_{n}^{-}\left(\mathscr{S}_{n}^{+}\right)$, we have $\alpha_{1} \geq \ldots \geq \alpha_{n}$ and $\alpha_{1}^{*} \geq \ldots \geq \alpha_{n}^{*}$. The hazard rate function of $Y_{1: n}$ can be written as the below:

$$
r_{Y_{1: n}}(x)=\left(1-\prod_{i=1}^{n} p_{i}\right) \mathrm{I}_{[x=0]}+\sum_{i=1}^{n} r\left(x ; \alpha_{i}, \beta_{i}\right) \mathrm{I}_{[x>0]},
$$

where $\mathrm{I}_{[.]}$denotes the indicator function. First, suppose that $x=0$. Clearly, $\prod_{i=1}^{n} p_{i}^{*} \leq \prod_{i=1}^{n} p_{i}$ implies that $r_{Y_{1: n}}(0) \leq r_{Y_{1: n}^{*}}(0)$. Second, suppose that $x>0$. In this case, let

$$
r_{Y_{1: n}}(x)=\Psi(\boldsymbol{\alpha})
$$

where

$$
\Psi(\boldsymbol{\alpha})=\sum_{i=1}^{n} r\left(x ; \alpha_{i}, \beta_{i}\right)
$$

The partial derivative of $\Psi(\boldsymbol{\alpha})$ with respect to $\alpha_{i}$ is given by

$$
\frac{\partial \Psi(\boldsymbol{\alpha})}{\partial \alpha_{i}}=\frac{\partial r\left(x ; \alpha_{i}, \beta_{i}\right)}{\partial \alpha_{i}} \geq[\leq] 0,
$$

which the inequality is obtained by condition (i). Since $r(x ; \alpha, \beta)$ is convex in $\alpha$ and $\frac{\partial r(x ; \alpha, \beta)}{\partial \alpha}$ is decreasing (increasing) in $\beta$, thus for $1 \leq i<j \leq n$, we have

$$
\frac{\partial r\left(x ; \alpha_{i}, \beta_{i}\right)}{\partial \alpha_{i}} \geq \frac{\partial r\left(x ; \alpha_{j}, \beta_{i}\right)}{\partial \alpha_{j}} \geq \frac{\partial r\left(x ; \alpha_{j}, \beta_{j}\right)}{\partial \alpha_{j}} .
$$

Therefore, we immediately conclude that

$$
\frac{\partial \Psi(\boldsymbol{\alpha})}{\partial \alpha_{i}}-\frac{\partial \Psi(\boldsymbol{\alpha})}{\partial \alpha_{j}}=\frac{\partial r\left(x ; \alpha_{i}, \beta_{i}\right)}{\partial \alpha_{i}}-\frac{\partial r\left(x ; \alpha_{j}, \beta_{j}\right)}{\partial \alpha_{j}} \geq 0
$$

In the view of Lemma 1 , the condition $\left(\alpha_{1}, \ldots, \alpha_{n}\right) \preceq_{\mathrm{w}}[\underline{\mathrm{w}}]\left(\alpha_{1}^{*}, \ldots, \alpha_{n}^{*}\right)$ implies that

$$
\Psi(\boldsymbol{\alpha}) \leq \Psi\left(\boldsymbol{\alpha}^{*}\right),
$$

which completes the proof. 
The proportional hazard rate (PHR) model and the scale model are very important models that has been extensively used in reliability theory, actuarial science and the other fields; see for example Cox (1972), Finkelstein (2008), Kumar and Klefsjö (1994), Balakrishnan et al. (2018), Nelson (1990) and Barmalzan et al. (2017). The both models are special cases of a model called the exponentiated scale model. A random variable $X$ is said to follow the exponentiated scale model, if its survival function can be written as

$$
\bar{F}(x ; \alpha, \beta)=[\bar{F}(\beta x)]^{\alpha}, \quad \alpha, \beta>0,
$$

where $\bar{F}(x)$ is a baseline survival function. The exponentiated scale model reduces to the PHR model if $\beta=1$ and it reduces to the scale model if $\alpha=1$.

The three following theorems provide comparisons between the smallest claim amounts in two independent heterogeneous portfolios of risks, whenever the severities follow the exponentiated scale model.

The following result deals with the comparison of the smallest claim amounts in two portfolios of risks, in the sense of the hazard rate ordering based on the comparison of $\boldsymbol{\alpha}$ and $\boldsymbol{\alpha}^{*}$ via the weak submajorization. It discusses the impact of the geometric mean of the occurrence probabilities and change of the parameters, on the smallest claim amounts. We find out that more geometric mean of the occurrence probabilities and less parameter vector in the sense of weak submajorization imply more minimum amount of the potential claims.

Theorem 2. Let $\bar{F}\left(x ; \alpha_{i}, \beta_{i}\right)=\left[\bar{F}\left(\beta_{i} x\right)\right]^{\alpha_{i}}$ and $\bar{F}\left(x ; \alpha_{i}^{*}, \beta_{i}\right)=\left[\bar{F}\left(\beta_{i} x\right)\right]^{\alpha_{i}^{*}}$, for $i=1, \ldots, n$. Under the setup of Theorem 1, suppose that the function $x r(x)$ is decreasing (increasing) in $x$. Then, for $(\boldsymbol{\alpha}, \boldsymbol{\beta}) \in \mathscr{S}_{n}^{-}\left(\mathscr{S}_{n}^{+}\right)$and $\left(\boldsymbol{\alpha}^{*}, \boldsymbol{\beta}\right) \in \mathscr{S}_{n}^{-}\left(\mathscr{S}_{n}^{+}\right)$, we have

$$
\prod_{i=1}^{n} p_{i}^{*} \leq \prod_{i=1}^{n} p_{i} \text { and }\left(\alpha_{1}, \ldots, \alpha_{n}\right) \preceq_{\mathrm{w}}\left(\alpha_{1}^{*}, \ldots, \alpha_{n}^{*}\right) \Longrightarrow Y_{1: n}^{*} \leq_{\mathrm{hr}} Y_{1: n} .
$$

Proof. Clearly, we have $r(x ; \alpha, \beta)=\alpha \beta r(\beta x)$, where $r(x)$ denotes the hazard function corresponding to the baseline distribution. It is easily obtained that $r(x ; \alpha, \beta)$ holds the condition (i) of Theorem 1. Moreover, the decreasingness (increasingness) property of $x r(x)$ implies the condition (ii) of Theorem 1 . Thus, the required result is obtained.

The next theorem provides a comparison of the smallest claim amounts in two portfolios of risks, in the sense of the hazard rate ordering based on the comparison of $\boldsymbol{\beta}$ and $\boldsymbol{\beta}^{*}$ via the weak submajorization. It evaluates the impact of the geometric mean of the occurrence probabilities and change of the parameters, on the smallest claim amounts. It is observed that more geometric mean of the occurrence probabilities and less parameter vactor in the sense of weak submajorization imply more the potential smallest claim amounts.

Theorem 3. Let $\bar{F}\left(x ; \alpha_{i}, \beta_{i}\right)=\left[\bar{F}\left(\beta_{i} x\right)\right]^{\alpha_{i}}$ and $\bar{F}\left(x ; \alpha_{i}, \beta_{i}^{*}\right)=\left[\bar{F}\left(\beta_{i}^{*} x\right)\right]^{\alpha_{i}}$, for $i=1, \ldots, n$. Under the setup of Theorem 1, suppose that the function $\operatorname{xr}(x)$ is increasing and convex in $x$. Then, for $(\boldsymbol{\alpha}, \boldsymbol{\beta}) \in \mathscr{S}_{n}^{+}$and $\left(\boldsymbol{\alpha}, \boldsymbol{\beta}^{*}\right) \in \mathscr{S}_{n}^{+}$, we have

$$
\prod_{i=1}^{n} p_{i}^{*} \leq \prod_{i=1}^{n} p_{i} \text { and }\left(\beta_{1}, \ldots, \beta_{n}\right) \preceq_{\mathrm{w}}\left(\beta_{1}^{*}, \ldots, \beta_{n}^{*}\right) \Longrightarrow Y_{1: n}^{*} \leq_{\mathrm{hr}} Y_{1: n} .
$$

Proof. By interchanging $\alpha$ and $\beta$ in Theorem 1 and some simple algebraic computations, it is easily seen that the conditions of Theorem 1 is derived under the assumptions of this Theorem on the function $\operatorname{xr}(x)$. Hence, the proof is completed.

Remark 1. Note that Theorem 3 includes the scale model when $\alpha_{i}=1, i=1, \ldots, n$, as a special case. Thus, we conclude Theorem 4 of Barmalzan et al. (2017). 
The following theorem provides a comparison between the smallest claim amounts in two portfolios of risks, in the sense of the hazard rate ordering based on the comparison of the parameter matrices $(\boldsymbol{\alpha}, \boldsymbol{\beta})$ and $\left(\boldsymbol{\alpha}^{*}, \boldsymbol{\beta}^{*}\right)$ via the row weak submajorization. It discusses the impact of the geometric mean of the occurrence probabilities and change of the parameters, on the smallest claim amounts.

Theorem 4. Let $\bar{F}\left(x ; \alpha_{i}, \beta_{i}\right)=\left[\bar{F}\left(\beta_{i} x\right)\right]^{\alpha_{i}}$ and $\bar{F}\left(x ; \alpha_{i}^{*}, \beta_{i}^{*}\right)=\left[\bar{F}\left(\beta_{i}^{*} x\right)\right]^{\alpha_{i}^{*}}$, for $i=1, \ldots, n$. Under the setup of Theorem 1, suppose that the function $\operatorname{xr}(x)$ is increasing and convex in $x$. Then, for $(\boldsymbol{\alpha}, \boldsymbol{\beta}) \in \mathscr{S}_{n}^{+}$and $\left(\boldsymbol{\alpha}^{*}, \boldsymbol{\beta}^{*}\right) \in \mathscr{S}_{n}^{+}$, we have

$$
\prod_{i=1}^{n} p_{i}^{*} \leq \prod_{i=1}^{n} p_{i} \text { and }\left[\begin{array}{c}
\alpha_{1} \ldots \alpha_{n} \\
\beta_{1} \ldots
\end{array} \beta_{n}\right]_{\mathrm{w}}\left[\begin{array}{c}
\alpha_{1}^{*} \ldots \alpha_{n}^{*} \\
\beta_{1}^{*} \ldots \beta_{n}^{*}
\end{array}\right] \Longrightarrow Y_{1: n}^{*} \leq_{\mathrm{hr}} Y_{1: n} .
$$

Proof. Let $V_{1: n}, Z_{1: n}$ and $W_{1: n}$ be the smallest claim amounts from the portfolios

$$
\begin{gathered}
\left(I_{p_{1}^{*}} X_{\alpha_{1: n}^{*}, \beta_{1: n}^{*}}, \ldots, I_{p_{n}^{*}} X_{\alpha_{n: n}^{*}, \beta_{n: n}^{*}}\right), \\
\left(I_{p_{1}^{*}} X_{\alpha_{1: n}, \beta_{1: n}^{*}}, \ldots, I_{p_{n}^{*}} X_{\alpha_{n: n}, \beta_{n: n}^{*}}\right), \\
\left(I_{p_{1}} X_{\alpha_{1: n}, \beta_{1: n}}, \ldots, I_{p_{n}} X_{\alpha_{n: n}, \beta_{n: n}}\right),
\end{gathered}
$$

respectively. It can be verified that $Y_{1: n}^{*} \stackrel{\text { hr }}{=} V_{1: n}$ and $Y_{1: n} \stackrel{\text { hr }}{=} W_{1: n}$. On the other hand, Theorem 2 and Theorem 3 imply that $V_{1: n} \leq_{\mathrm{hr}} Z_{1: n}$ and $Z_{1: n} \leq_{\mathrm{hr}} W_{1: n}$, respectively. Hence, the required result is obtained.

Since the weak submajorization is implied by the chain majorization, the majorization and the row majorization, thus the result of Theorem 4 is also obtained under these majorizations.

Burr distribution is a useful distribution to depict the claim amounts. This distribution first introduced by Singh and Maddala (1975); while it seems that it was first considered by Burr (1942). To observe some applications of this distribution in actuary, one may refer to Hogg and Klugman (1983) and Cummins, Dionne, McDonald, and Pritchett (1990). Also, for more details, one may refer to Hogg and Klugman (1984) and Kleiber and Kotz (2003). A random variable $X$ has the Burr distribution with the shape parameters $\alpha$ and $\lambda$ and the rate parameter $\beta$, denoted by $X \sim \operatorname{Burr}(\alpha, \beta, \lambda)$, if its survival function is given by

$$
\bar{F}(x ; \alpha, \beta, \lambda)=\left(1+(\beta x)^{\lambda}\right)^{-\alpha}, \quad x>0, \alpha, \beta, \lambda>0,
$$

where $\alpha$ only affects the right tail, whereas $\lambda$ affects both tails. Clearly, the Lomax distribution which was introduced by Lomax (1954) is a special case of the Burr distribution for $\lambda=1$.

The following theorem assumes the Burr distribution as the distribution of the severities in two portfolios of risks and provides a comparison of the smallest claim amounts between two portfolios.

Theorem 5. Let $X_{\alpha_{i}} \sim \operatorname{Burr}\left(\alpha_{i}, \beta_{i}, \lambda\right)$ and $X_{\alpha_{i}^{*}} \sim \operatorname{Burr}\left(\alpha_{i}^{*}, \beta_{i}, \lambda\right)$, for $i=1, \ldots, n$. Under the setup of Theorem 3, for $(\boldsymbol{\alpha}, \boldsymbol{\beta}) \in \mathscr{S}_{n}^{+}$and $\left(\boldsymbol{\alpha}^{*}, \boldsymbol{\beta}\right) \in \mathscr{S}_{n}^{+}$and any $\lambda>0$, we have

$$
\prod_{i=1}^{n} p_{i}^{*} \leq \prod_{i=1}^{n} p_{i} \text { and }\left(\alpha_{1}, \ldots, \alpha_{n}\right) \preceq_{\mathrm{w}}\left(\alpha_{1}^{*}, \ldots, \alpha_{n}^{*}\right) \Longrightarrow Y_{1: n}^{*} \leq_{\mathrm{hr}} Y_{1: n} .
$$

Proof. It can be easily verified that $x r(x)=\frac{\lambda x^{\lambda}}{1+x^{\lambda}}$ is increasing in $x$. Hence, Theorem 2 immediately completes the proof.

The following example provides a numerical example to illustrate the validity of Theorem 5 . 
Example 1. Let $X_{\alpha_{i}} \sim \operatorname{Burr}\left(\alpha_{i}, \beta_{i}, 2\right)\left(X_{\alpha_{i}^{*}} \sim \operatorname{Burr}\left(\alpha_{i}^{*}, \beta_{i}, 2\right)\right)$, for $i=1,2,3$. Further, suppose that $I_{p_{1}}, I_{p_{2}}, I_{p_{3}}\left(I_{p_{1}^{*}}, I_{p_{2}^{*}}, I_{p_{3}^{*}}\right)$ is a set of independent Bernoulli random variables, independent of the $X_{\alpha_{i}}$ 's $\left(X_{\alpha_{i}^{*}}\right.$ 's $)$, with $\mathrm{E}\left[I_{p_{i}}\right]=p_{i}\left(\mathrm{E}\left[I_{p_{i}^{*}}\right]=p_{i}^{*}\right)$, for $i=1,2,3$. We take $\left(\alpha_{1}, \alpha_{2}, \alpha_{3}\right)=$ $(4,0.7,0.2),\left(\alpha_{1}^{*}, \alpha_{2}^{*}, \alpha_{3}^{*}\right)=(4.2,0.6,0.3),\left(\beta_{1}, \beta_{2}, \beta_{3}\right)=(5,3.5,1.2),\left(p_{1}, p_{2}, p_{3}\right)=(0.2,0.3,0.4)$, and $\left(p_{1}^{*}, p_{2}^{*}, p_{3}^{*}\right)=(0.25,0.35,0.25)$. It is clear that the conditions of Theorem 5 are satisfied. So, we have $Y_{1: n}^{*} \leq_{\mathrm{hr}} Y_{1: n}$. Figure 1 represents the function $r_{Y_{1: n}^{*}}(x)-r_{Y_{1: n}}(x)$, which agrees with the intended result.

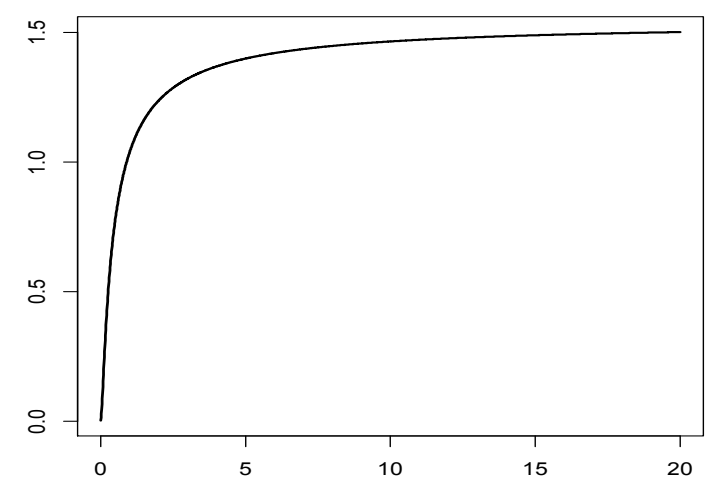

Figure 1: $\quad$ Plot of the function $r_{Y_{1: n}^{*}}(x)-r_{Y_{1: n}}(x)$ corresponding to Example 1.

The following example illustrates that the conditions $(\boldsymbol{\alpha}, \boldsymbol{\beta}) \in \mathscr{S}_{n}^{+}$and $\left(\boldsymbol{\alpha}^{*}, \boldsymbol{\beta}\right) \in \mathscr{S}_{n}^{+}$are the important conditions and can not be dropped.

Example 2. Under the same setup in Example 1, we take $\left(\alpha_{1}^{*}, \alpha_{2}^{*}, \alpha_{3}^{*}\right)=(4.2,0.3,0.6)$ with the other unchanged values. It is clear that $\left(\boldsymbol{\alpha}^{*}, \boldsymbol{\beta}\right) \notin \mathscr{S}_{n}^{+}$, but it can be easily verified that the other conditions of Theorem 5 are satisfied. Figure 2 represents the function $r_{Y_{1: n}^{*}}(x)-r_{Y_{1: n}}(x)$, which changes the sign.

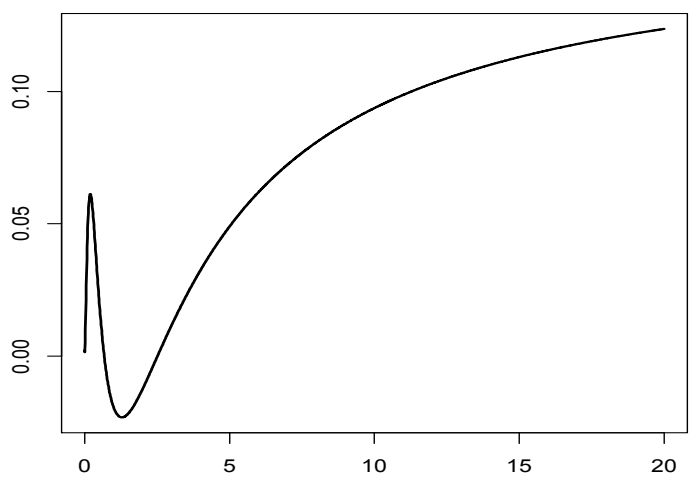

Figure 2: $\quad$ Plot of the function $r_{Y_{1: n}^{*}}(x)-r_{Y_{1: n}}(x)$ corresponding to Example 2.

Barmalzan et al. (2018) considered the Marshall-Olkin extended exponential distribution as the claim amount distribution in portfolios of risks and compared the aggregate claim amounts in two heterogeneous portfolios. Marshall-Olkin distribution, which was introduced by Marshall and Olkin (1997) is a spacial case of a wide range family of distributions, called Harris family. Aly and Benkherouf (2011) used the Harris distribution which has been introduced by Harris (1948), and generated the Harris family. A random variable $X$ is said to follow the Harris family, denoted by $X \sim \operatorname{Harris}(\alpha, \beta)$, if its survival function is given by

$$
\bar{F}(x ; \alpha, \beta)=\left(\frac{\beta \bar{F}^{\alpha}(x)}{1-(1-\beta) \bar{F}^{\alpha}(x)}\right)^{1 / \alpha}, \quad \alpha, \beta>0,
$$


where $\bar{F}(x)$ is a baseline survival function.

The following theorem provides a comparison of the smallest claim amounts in two heterogeneous portfolios of risks by assuming that the severities in two portfolios belong to the Harris family.

Theorem 6. Let $X_{\alpha_{i}} \sim \operatorname{Harris}\left(\alpha_{i}, \beta_{i}\right)$ and $X_{\alpha_{i}^{*}} \sim \operatorname{Harris}\left(\alpha_{i}^{*}, \beta_{i}\right)$, for $i=1, \ldots, n$. Further assume that $0<\beta_{i} \leq 1$, for $i=1, \ldots, n$. Under the setup of Theorem 1 , for $(\boldsymbol{\alpha}, \boldsymbol{\beta}) \in \mathscr{S}_{n}^{+}$ and $\left(\boldsymbol{\alpha}^{*}, \boldsymbol{\beta}\right) \in \mathscr{S}_{n}^{+}$, we have

$$
\prod_{i=1}^{n} p_{i}^{*} \leq \prod_{i=1}^{n} p_{i} \text { and }\left(\alpha_{1}, \ldots, \alpha_{n}\right) \stackrel{\mathrm{w}}{\preceq}\left(\alpha_{1}^{*}, \ldots, \alpha_{n}^{*}\right) \Longrightarrow Y_{1: n}^{*} \leq \mathrm{hr} Y_{1: n} .
$$

Proof. Obviously, we have $r(x ; \alpha, \beta)=\frac{r(x)}{1-(1-\beta) \bar{F}^{\alpha}(x)}$. It can be verified that $r(x ; \alpha, \beta)$ imply the condition (i) of Theorem 1. Moreover, the assumption $0<\beta \leq 1$ implies the condition (ii) of Theorem 1. Thus, the proof is completed.

The following example gives a numerical example to illustrate the validity of Theorem 6 .

Example 3. Let $X_{\alpha_{i}} \sim \operatorname{Harris}\left(\alpha_{i}, \beta_{i}\right)\left(X_{\alpha_{i}^{*}} \sim \operatorname{Harris}\left(\alpha_{i}^{*}, \beta_{i}\right)\right)$, for $i=1,2,3$, with the baseline gamma distribution with the shape parameter 2 and the scale parameter 1 in each two sets. Further, suppose that $I_{p_{1}}, I_{p_{2}}, I_{p_{3}}\left(I_{p_{1}^{*}}, I_{p_{2}^{*}}, I_{p_{3}^{*}}\right)$ is a set of independent Bernoulli random variables, independent of the $X_{\alpha_{i}}$ 's $\left(X_{\alpha_{i}^{*}}\right.$ 's), with $\mathrm{E}\left[I_{p_{i}}\right]=p_{i}\left(\mathrm{E}\left[I_{p_{i}^{*}}\right]=p_{i}^{*}\right)$, for $i=1,2,3$. We take $\left(\alpha_{1}, \alpha_{2}, \alpha_{3}\right)=(1.1,1.6,2.5),\left(\alpha_{1}^{*}, \alpha_{2}^{*}, \alpha_{3}^{*}\right)=(1,1.7,2.3),\left(\beta_{1}, \beta_{2}, \beta_{3}\right)=(0.1,0.3,0.6)$, $\left(p_{1}, p_{2}, p_{3}\right)=(0.1,0.05,0.15)$, and $\left(p_{1}^{*}, p_{2}^{*}, p_{3}^{*}\right)=(0.2,0.05,0.05)$. It is clear that the conditions of Theorem 6 are satisfied. So, we have $Y_{1: n}^{*} \leq_{\mathrm{hr}} Y_{1: n}$. Figure 3 represents the function $r_{Y_{1: n}^{*}}(x)-r_{Y_{1: n}}(x)$, which coincides with the intended result.

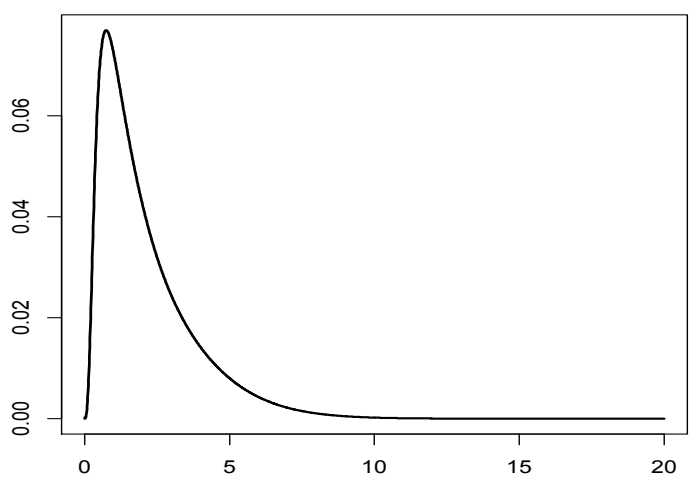

Figure 3: Plot of the function $r_{Y_{1: n}^{*}}(x)-r_{Y_{1: n}}(x)$ corresponding to Example 3.

\section{Conclusion}

We know that the smallest and largest claim amounts provide useful informations for the insurance companies to determine the annual premium. Therefore, we consider the smallest claim amounts to study. In this paper, under some conditions, we studied the stochastic comparisons between the smallest claim amounts in the sense of the hazard rate ordering, when the severities are independent and heterogeneous, and they belonging to a general model. Based on the published articles in this field, it is known that the scale and PHR models are very applicable in insurance analysis and actuary. In continuation of our discussion, we investigated the case that the severities belong to the exponentiated scale model which includes the both scale model and the PHR model. In this model and under some certain conditions, we observed that more geometric mean of the occurrence probabilities and less 
parameter matrix in the sense of row weak submajorization, potentially imply more smallest amount of the claims. Then we considered the Burr distribution and the Harris family as a model for the severities and compared the smallest claim amounts. These results can provide useful perspective for the insurance companies in order to determine the annual premiums. It is necessary to mention that all results of this paper are also valid for comparison of series systems in the sense of the hazard rate ordering when the starting random shocks may impact the components.

As a possible generalization, it will be of interest to investigate the ordering properties of the smallest claim amounts when each portfolio be interdependent; i.e. the existing severities in the portfolio are dependent. We are working on this problem and hope to present our findings in a future paper.

\section{Acknowledgements}

We would like to express our sincere thanks to the valued reviewer and editor for their constructive suggestions and comments that helped us to improve the manuscript.

\section{References}

Aly EEAA, Benkherouf L (2011). "A New Family of Distributions Based on Probability Generating Functions." Sankhya B, 73(1), 70-80. doi:10.1007/s13571-011-0017-9.

Balakrishnan N, Zhang Y, Zhao P (2018). "Ordering the Largest Claim Amounts and Ranges from Two Sets of Heterogeneous Portfolios." Scandinavian Actuarial Journal, 2018(1), 23-41. doi:10.1080/03461238.2017.1278717.

Barmalzan G, Najafabadi ATP (2015). "On the Convex Transform and Right-spread Orders of Smallest Claim Amounts." Insurance: Mathematics and Economics, 64, 380-384. doi: $10.1016 / j$.insmatheco.2015.07.001.

Barmalzan G, Najafabadi ATP, Balakrishnan N (2015). "Stochastic Comparison of Aggregate Claim Amounts between Two Heterogeneous Portfolios and Its Applications." Insurance: Mathematics and Economics, 61, 235-241. doi:10.1016/j.insmatheco.2015.01.010.

Barmalzan G, Najafabadi ATP, Balakrishnan N (2016). "Likelihood Ratio and Dispersive Orders for Smallest Order Statistics and Smallest Claim Amounts from Heterogeneous Weibull Sample." Statistics \& Probability Letters, 110, 1-7. doi:10.1016/j .spl.2015.11. 009.

Barmalzan G, Najafabadi ATP, Balakrishnan N (2017). "Ordering Properties of the Smallest and Largest Claim Amounts in a General Scale Model." Scandinavian Actuarial Journal, 2017(2), 105-124. doi:10.1080/03461238.2015.1090476.

Barmalzan G, Najafabadi ATP, Balakrishnan N (2018). "Some New Results on Aggregate Claim Amounts from Two Heterogeneous Marshall-Olkin Extended Exponential Portfolios." Communications in Statistics-Theory and Methods, 47(11), 2779-2794. doi: 10.1080/03610926.2017.1343844.

Burr IW (1942). "Cumulative Frequency Functions." The Annals of Mathematical Statistics, $\mathbf{1 3}(2), 215-232$.

Cox DR (1972). "Regression Models and Life Tables." Journal of the Royal Statistical Society: Series B (Methodological), 34(2), 187-202. doi:10.1111/j.2517-6161.1972.tb00899.x. 
Cummins JD, Dionne G, McDonald JB, Pritchett BM (1990). "Applications of the GB2 Family of Distributions in Modeling Insurance Loss Processes." Insurance: Mathematics and Economics, 9(4), 257-272. doi:10.1016/0167-6687(90)90003-V.

Denuit M, Frostig E (2006). "Heterogeneity and the Need for Capital in the Individual Model." Scandinavian Actuarial Journal, 2006(1), 42-66. doi:10.1080/03461230500518690.

Finkelstein M (2008). Failure Rate Modeling for Reliability and Risk. Springer, London.

Frostig E (2001). "A Comparison between Homogeneous and Heterogeneous Portfolios." Insurance: Mathematics and Economics, 29(1), 59-71. doi:10.1016/S0167-6687(01)00073-7.

Harris TE (1948). "Branching Processes." The Annals of Mathematical Statistics, 19(4), $474-494$.

Hogg RV, Klugman SA (1983). "On the Estimation of Long Tailed Skewed Distributions with Actuarial Applications." Journal of Econometrics, 23(1), 91-102. doi:10.1016/ 0304-4076 (83) 90077-5.

Hogg RV, Klugman SA (1984). Loss Distributions. John Wiley \& Sons, New York.

Hu T, Ruan L (2004). "A Note on Multivariate Stochastic Comparisons of Bernoulli Random Variables." Journal of Statistical Planning and Inference, 126(1), 281-288. doi:10.1016/ j.jspi.2003.07.012.

Karlin S, Novikoff A (1963). "Generalized Convex Inequalities." Pacific Journal of Mathematics, 13(4), 1251-1279.

Khaledi BE, Ahmadi SS (2008). "On Stochastic Comparison between Aggregate Claim Amounts." Journal of Statistical Planning and Inference, 138(7), 2243-2251. doi: 10.1016/j.jspi.2007.10.018.

Kleiber C, Kotz S (2003). Statistical Size Distributions in Economics and Actuarial Sciences. John Wiley \& Sons, New Jersey.

Kumar D, Klefsjö B (1994). "Proportional Hazards Model: a Review." Reliability Engineering \& System Safety, 44(2), 177-188. doi:10.1016/0951-8320(94)90010-8.

Li C, Li X (2016). "Sufficient Conditions for Ordering Aggregate Heterogeneous Random Claim Amounts." Insurance: Mathematics and Economics, 70, 406-413. doi:10.1016/j. insmatheco.2016.07.008.

Li H, Li X (2013). Stochastic Orders in Reliability and Risk. Springer, New York.

Lomax KS (1954). "Business Failures: Another Example of the Analysis of Failure Data." Journal of the American Statistical Association, 49(268), 847-852. doi:10.1080/ 01621459.1954.10501239.

Ma C (2000). "Convex Orders for Linear Combinations of Random Variables." Journal of Statistical Planning and Inference, 84, 11-25. doi:10.1016/S0378-3758(99)00143-3.

Marshall AW, Olkin I (1997). "A New Method for Adding a Parameter to a Family of Distributions with Application to the Exponential and Weibull Families." Biometrika, 84(3), 641-652. doi:10.1093/biomet/84.3.641.

Marshall AW, Olkin I, Arnold BC (2011). Inequalities: Theory of Majorization and Its Applications. Springer, New York.

Müller A, Stoyan D (2002). Comparison Methods for Stochastic Models and Risks. John Wiley \& Sons, New York. 
Nadeb H, Torabi H, Dolati A (2020a). "Stochastic Comparisons between the Extreme Claim Amounts from Two Heterogeneous Portfolios in the Case of Transmuted-G Model." North American Actuarial Journal. doi:10.1080/10920277.2019.1671203.

Nadeb H, Torabi H, Dolati A (2020b). "Stochastic Comparisons of the Largest Claim Amounts from Two Sets of Interdependent Heterogeneous Portfolios." Mathematical Inequalities \& Applications, 23(1), 35-56. doi:10.7153/mia-2020-23-03.

Nelson W (1990). Accelerated Testing, Statistical Models, Test Plans and Data Analysis. John Wiley \& Sons, New Jersey.

Shaked M, Shanthikumar JG (2007). Stochastic Orders. Springer, New York.

Singh SK, Maddala GS (1975). "A Stochastic Process for Income Distribution and Tests for Income Distribution Functions." In ASA Proceedings of the Business and Economic Statistics Section, pp. 551-553.

Zhang Y, Zhao P (2015). "Comparisons on Aggregate Risks from Two Sets of Heterogeneous Portfolios." Insurance: Mathematics and Economics, 65, 124-135. doi: $10.1016 / j$. insmatheco.2015.09.004.

\section{Affiliation:}

Hossein Nadeb

Department of Statistics

Yazd University

Yazd, Iran

E-mail: honadeb@yahoo.com

Hamzeh Torabi

Department of Statistics

Yazd University

Yazd, Iran

E-mail: htorabi@yazd.ac.ir

\section{Austrian Journal of Statistics}

published by the Austrian Society of Statistics

Volume 50

July 2021 http://www.ajs.or.at/

http://www.osg.or.at/

Submitted: 2019-09-06

Accepted: 2020-01-26 